\title{
Efecto de la radiación UV en arcillas expansivas tratadas con aceite sulfonado
}

\author{
Javier F. Camacho-Tauta", Oscar J. Reyes-Ortiz", Catalina Mayorga* \\ * Programa de Ingeniería Civil, Universidad Militar Nueva Granada \\ §javier.camacho@unimilitar.edu.co
}

(Recibido: Octubre 27 de 2009- Aceptado: Noviembre 22 de 2010)

\begin{abstract}
Resumen
La estabilización de arcillas expansivas con aceite sulfonado es un proceso electroquímico que se desarrolla a lo largo del tiempo y cuyo progreso depende de las condiciones ambientales existentes en el campo. La evolución de este fenómeno se estudió mediante el análisis experimental de tres tipos de arcilla expansiva sometidas a tratamiento con aceite sulfonado en diferentes dosificaciones. Para simular las condiciones ambientales, estas arcillas fueron expuestas a radiación UV en una cámara de envejecimiento acelerado. Una vez conseguido el periodo de envejecimiento programado, se realizaron ensayos de límites de consistencia a las muestras para determinar la influencia del envejecimiento y el porcentaje de aceite sulfonado en las propiedades expansivas de las arcillas. Los resultados obtenidos permitieron observar que la plasticidad del material depende del periodo de exposición y la dosificación de aceite sulfonado utilizada.
\end{abstract}

Palabras Claves: Envejecimiento acelerado, Arcilla expansiva, Potencial de expansión, Límites de consistencia, Aceite sulfonado, Radiación UV, Radiación solar.

CIVIL ENGINEERING

\section{Effect of UV radiation in expansive clays with sulfonade oil}

\begin{abstract}
Stabilization of expansive clays with sulfonated oil is an electrochemical process that develops over time and whose progress depends on the environmental conditions in the field. This phenomenon was studied by mean of an experimental analysis of three types of expansive clay treated with sulfonated oil. To simulate the environmental conditions, these clays were subjected to UV radiation in an accelerated aging chamber. Once obtained the programmed aging period, plasticity limits of soils allowed determining the influence of aging and concentration of sulfonated oil on the properties of expansive clays. Results revealed that the final plasticity and hence the swelling potential of the material depends on the period of exposure and the concentration of sulfonated oil. Finally, it is suggested to adopt the methodology used in this study to determine the concentration of sulfonated oil, which is a function of the type of clay.
\end{abstract}

Keywords: Accelerated aging, Expansive clay, Swelling potential, Plasticity limits, Sulfonated oil, UV radiation, Solar radiation 


\section{Introducción}

Los suelos expansivos presentan un comportamiento perjudicial para cualquier estructura construida sobre estos, porque tienen un comportamiento caracterizado por su variación volumétrica ante la presencia de agua y su posterior reducción. La actividad eléctrica de los cationes que los componen, crean un enlace electroquímico arcilla-agua y forman una capa de agua en el entorno de cada partícula del material, lo que ocasiona variaciones volumétricas que inducen esfuerzos adicionales a las estructuras construidas sobre este tipo de arcillas (Química Cros, 2007).

Múltiples investigadores han realizado estudios en suelos expansivos para determinar el comportamiento de sus propiedades ante la exposición natural de ciclos de humedecimiento y secado. Algunos, resultados observados muestran que para el primer ciclo de humedecimiento y secado, la cantidad de expansión vertical es pequeña, pero en los posteriores ciclos, la magnitud aumenta significativamente (Day, 1994). Por otra parte, se ha observado que el hinchamiento inicial depende de la magnitud relativa del contenido de humedad con relación a la humedad óptima de compactación. Así, si el material se compacta del lado seco de la curva, el hinchamiento producido durante el primer ciclo será mayor (Gens y Alonso, 1992). Estos cambios sugieren que es necesario estudiar mayores periodos de exposición para poder encontrar resultados más concluyentes, si se tiene en cuenta que las reacciones foto-químicas, normalmente no son reacciones simples de un paso (Gadomski, 2007).

Resultados obtenidos por Kassiff y Baker (1971), sugieren que las arcillas expansivas pueden compactarse a la densidad seca óptima, siempre y cuando se le de suficiente tiempo para el proceso de envejecimiento y que para dicha densidad seca, la cantidad de potencial de expansión no sea afectada por la humedad inicial.

Subba y Tripathy (2003) estudiaron los efectos del envejecimiento en la expansión y contracción de un suelo expansivo compactado, determinando que los factores dominantes que influyen en los efectos del envejecimiento son: el contenido de humedad y el grado de saturación al comienzo del proceso de envejecimiento. Mishra et al. (2007) afirman que el potencial de expansión de un suelo está influenciado por dos factores; el primero que depende de la naturaleza de las partículas del suelo y el segundo, del estado físico y ambiental del suelo, que incluye la densidad seca y el contenido de agua en la compactación.

Arellana (2004), mostró que el efecto estabilizador del aceite sulfonado sobre una arcilla expansiva, depende de las condiciones ambientales y que se desarrolla a lo largo del tiempo, por lo que se hace necesario estudiar su evolución mediante el análisis de especímenes sometidos a diferentes periodos de curado. Lo anterior hace que un estudio sistemático de este fenómeno, requiera de tiempos excesivos especialmente cuando las condiciones ambientales son las menos propicias para el desarrollo del efecto estabilizador del aceite sulfonado (Camacho et al., 2006). Es por esto, que el objetivo principal de esta investigación esta relacionada en determinar el efecto en las propiedades expansivas de una arcilla por la adición de aceite sulfonado y la incidencia del envejecimiento acelerado mediante una cámara de radiación $U V$.

\section{Marco conceptual}

Los aceites sulfonados son agentes catalizadores que producen intercambio de iones; químicamente son compuestos orgánicos derivados de sulfuros y ácidos combinados. La función más importante de estos aceites es la reducción del agua contenida entre las partículas del suelo, aumentando el número de vacíos que permiten el reacomodamiento de las partículas, bien sea por atracción entre ellas o por compactación (Páez, 2005).

El aceite sulfonado es un líquido soluble en agua, a la que ioniza aumentando su conductividad y facilitando el intercambio catiónico, ya que la obliga a desprenderse de las partículas de arcilla y la convierte en agua libre, capaz de intercambiar sus cargas eléctricas con los cationes de la arcilla, a los que finalmente neutraliza y obliga en forma permanente a perder su avidez de agua.

Los principales efectos que tiene el aceite sulfonado sobre los suelos de matriz arcillosa son: 
reduce espacios intersticiales, reduce la permeabilidad, incrementa la sedimentación, mejora la respuesta a la compactación y aumenta la densidad del suelo. Los estudios realizados con aceites sulfonados y la evidencia obtenida mediante ensayos realizados en campo, muestran que el sistema de estabilización electroquímica es una alternativa competitiva para disminuir el potencial expansivo de los suelos arcillosos (Arellana, 2004; Garzón et al., 2004; Rauch et al., 2003). No se han reportado efectos contaminantes por el uso de aceite sulfonado en las concentraciones utilizadas para esta aplicación.

La radiación solar es la energía emitida por el sol que se propaga en todas las direcciones a través del espacio mediante ondas electromagnéticas. $\mathrm{La}$ irradiancia (E) es una medida de la potencia o flujo de energía recibida $(\Phi)$ por unidad de área $(A)$, Ec. (1). La exposición radiante $(\mathrm{H})$ es una medida de la irradiancia acumulada a lo largo de un periodo de tiempo (t) que normalmente es un día, Ec. (2). (IDEAM 2005; Rodríguez y González, 1992).

$$
\begin{aligned}
& E=\frac{d \Phi}{d A} \\
& H=\int_{t_{1}}^{t_{2}} E d t
\end{aligned}
$$

Las cámaras de envejecimiento acelerado permiten lograr en menor tiempo los cambios que se presentan en un material por una exposición natural prolongada, porque simula condiciones de radiación $\mathrm{UV}$, temperatura y humedad relativa, y a su vez estas pueden controlarse, lo que garantiza la repetitividad y evaluación a diferentes tiempos de exposición. Teóricamente se puede determinar el tiempo necesario de exposición en cámara que simule una cantidad de irradiancia total acumulada correspondiente a la recibida en campo en un periodo de tiempo determinado.

La irradiancia aplicada mediante la cámara de envejecimiento acelerado $\mathrm{E}_{\mathrm{a}}$ utilizada en la investigación es igual a $1.6 \mathrm{~W} / \mathrm{m}^{2} . n m$ que a $340 \mathrm{~nm}$ equivale a $544 \mathrm{~W} / \mathrm{m}^{2}$. Para efectos de comparación, la irradiancia UV promedio en la ciudad de Bogotá $\left(E_{c}\right)$, se encuentra alrededor de $67 \mathrm{~W} / \mathrm{m}^{2}$ (IDEAM, 2009). Por consiguiente, igualando la exposición radiante en campo $\left(\mathrm{H}_{\mathrm{c}}\right)$, con la exposición radiante en cámara de envejecimiento acelerado $\left(\mathrm{H}_{\mathrm{a}}\right)$, se puede obtener el tiempo equivalente en cámara $\left(\mathrm{t}_{\mathrm{a}}\right)$ para obtener la misma irradiancia acumulada:

$$
\mathrm{t}_{\mathrm{a}}=\frac{\mathrm{H}_{\mathrm{c}}}{\mathrm{E}_{\mathrm{a}}}
$$

Visto de otro modo, la relación (k) entre el tiempo en campo $\left(\mathrm{t}_{\mathrm{c}}\right)$ y el tiempo en cámara $\left(\mathrm{t}_{\mathrm{a}}\right)$ que corresponden a la misma exposición radiante, para un periodo de tiempo, se puede determinar como:

$$
\mathrm{k}=\frac{\mathrm{E}_{a}}{\mathrm{E}_{\mathrm{c}}\left(\frac{\text { horas sol/día }}{24 \text { horas/día }}\right)}
$$

Este factor permite por consiguiente estimar el tiempo equivalente de curado en condiciones naturales, cuando un material se somete a exposición acelerada en una cámara de envejecimiento acelerado (Reyes y Camacho, 2008). Para el ejemplo citado de la ciudad de Bogotá, el factor $\mathrm{k}$ equivale a 16.24. Este factor puede variar dependiendo de la época del año que se esté simulando.

\section{Metodología}

Se analizaron tres tipos de arcilla con diferente potencial de expansión, obtenidas a partir de mezclas de bentonita sódica (IP $\leq 293 \%$, contenido de finos $\leq 75 \%$ ) disponible comercialmente (Bentocol Ltda.) y caolín (IP $\leq 20 \%$, contenido de finos $\leq 36 \%$ ), en las proporciones que se muestran en la Tabla 1. En la misma tabla se indican los límites de plasticidad, el peso unitario seco máximo y el contenido óptimo de humedad obtenidos del ensayo de compactación Proctor modificado (ASTM D 698).

A continuación a las mezclas se le adicionó aceite sulfonado (Geo-Stab producido por Anpatec Ltda.) 
Ingeniería y Competitividad, Volumen 12, No. 2, p. 41 - 50 (2010)

Tabla 1 Proporciones y caracterización inicial de la arcilla utilizada

\begin{tabular}{lllllll}
\hline Tipo & Bentonita(\%) & Caolín (\%) & LL $(\%)$ & IP $(\%)$ & $\gamma_{\mathrm{d}}\left(\mathrm{g} / \mathrm{cm}^{3}\right)$ & $\omega(\%)$ \\
\hline I & 6 & 94 & 50 & 30 & 1.61 & 18.1 \\
II & 15 & 85 & 73 & 53 & 1.61 & 21.0 \\
III & 23 & 77 & 110 & 89 & 1.58 & 16.4 \\
\hline
\end{tabular}

disuelto en agua en concentraciones de 25,50 y 100 centímetros cúbicos de aceite sulfonado por cada metro cúbico de material compactado.

Se mezclaron las muestras con las concentraciones de aceite sulfonado disueltas en la cantidad de agua correspondiente a la humedad óptima previamente. Teniendo en cuenta que la investigación se enmarcó dentro de las condiciones de radiación media para la ciudad de Bogotá, en la Tabla 2 se presentan los valores promedio.

Posteriormente, las muestras se expusieron a ciclos continuos de ocho horas de radiación UV$1.60 \mathrm{~W} / \mathrm{m}^{2}$ a $340 \mathrm{~nm}, 45^{\circ} \mathrm{C}$ y de cuatro horas de condensación a una humedad relativa del 100\%, para ensayarse a diferentes periodos de curado en la cámara de envejecimiento acelerado (Figura 1).

Cada muestra al cumplir el tiempo de curado previsto, se sometió a ensayos de plasticidad: límite líquido (LL), límite plástico (LP) e índice de plasticidad (IP) (INV E-125, E-126, E-127), que permiten estimar el potencial expansivo del suelo (Tabla 3). Para la realización de los ensayos de plasticidad se utilizó agua destilada. Aún cuando el proceso de ensayo implica alterar el contenido de humedad del material y por consiguiente la concentración de aceite sulfonado, se considera que éste procedimiento no tiene incidencia sobre el resultado, puesto que la corta duración del

Tabla 2 Valores promedio de variables de radiación para Bogotá (IDEAM, 2009).

\begin{tabular}{lll}
\hline Parámetro & Unidad & Valor medio \\
\hline Radiación solar global diaria & $\mathrm{kWh} / \mathrm{m}^{2}$ & 3.77 \\
Radiación UV-A diaria & $\mathrm{kWh} / \mathrm{m}^{2}$ & 0.81 \\
\hline
\end{tabular}

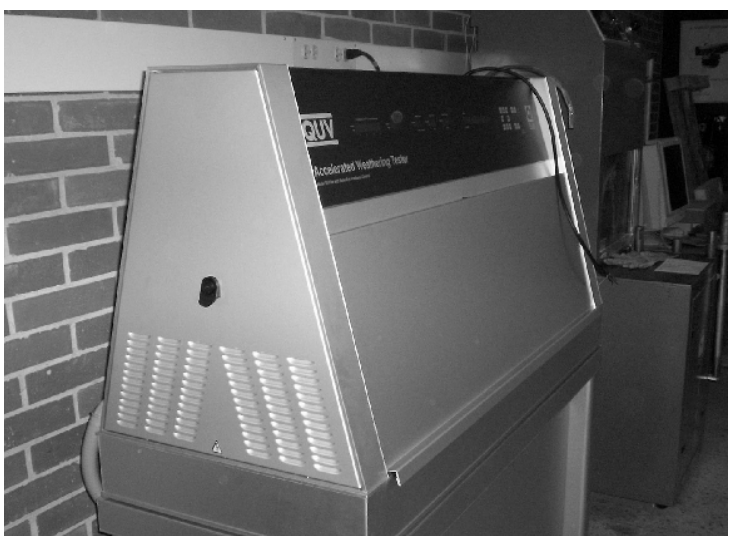

Figura 1. Cámara de envejecimiento acelerado por radiación UV. (U. Militar Nueva Granada) 
Tabla 3. Potencial Expansivo del Suelo. (INVIAS E-132)

\begin{tabular}{llll}
\hline Norma $\longrightarrow$ & INV E-125 & INV E-126 & $\begin{array}{l}\text { AASHTO T-273 } \\
\text { Succión del Suelo } \\
\left(\mathrm{kN} / \mathrm{m}^{2}\right)\end{array}$ \\
Grado de Expansión & LL \% & IP \% & $>422.4$ \\
Elevado & $>60$ & $<35$ & $158.4-422.4$ \\
Marginal & $50-60$ & $25-35$ & $<158.4$ \\
Bajo & $<50$ & $<25$ & \\
\hline
\end{tabular}

ensayo en condiciones de laboratorio no modifica los efectos ya producidos por la concentración inicial del aceite sulfonado en condiciones de envejecimiento acelerado.

Es conveniente anotar que la plasticidad del suelo es solamente un indicador del potencial de hinchamiento y que se utiliza en esta investigación para evaluar inicialmente el efecto del aceite sulfonado. Una investigación más rigurosa implica la medición directa del potencial de hinchamiento mediante métodos apropiados como el ensayo de expansión Lambe o el ensayo de expansión con sobre carga descritos en la Norma INV E-132.

\section{Análisis de resultados}

Se observa en las Figuras 2 y 3 la evolución de los límites líquido y el índice de plasticidad respectivamente, para la arcilla tipo I. Dado que el potencial de expansión inicial de este material es calificado como marginal, no se presenta una reducción apreciable en la plasticidad del material como efecto de la adición de aceite sulfonado. Ninguna de las dosificaciones utilizadas se aparta significativamente de la curva correspondiente al material sin tratar.

En la Figura 3, se repite la misma observación, salvo por una leve disminución de la plasticidad que se presenta para todas las dosificaciones incluido el material sin tratar. Puede verificarse que la clasificación del potencial expansivo, de acuerdo con los criterios indicados en la Tabla 3, pasa de ser marginal a bajo.

En la Figura 4, se presenta la variación del límite líquido para la arcilla tipo II, cuyo potencial de expansión es inicialmente elevado. En este caso es

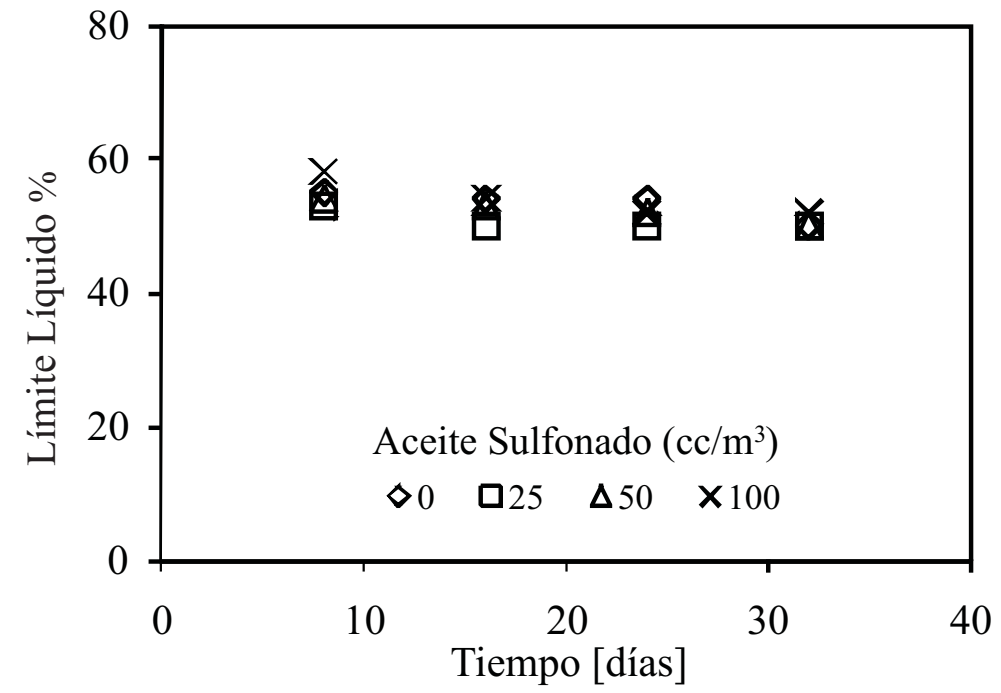

Figura 2. Variación del límite líquido con el tiempo de exposición, para la arcilla tipo I. 
bastante clara la diferencia entre el material no tratado y el tratado con aceite sulfonado. En el primer caso el límite líquido se mantiene prácticamente alrededor del valor inicial (73\%), mientras que en el segundo caso, la reducción en el límite líquido es apreciable, llegando a reducir el potencial de expansión hasta un nivel marginal.

En la Figura 5, se presenta la variación del índice de plasticidad de la arcilla tipo II y se corrobora que la mayor reducción se consigue con la dosificación correspondiente a $25 \mathrm{cc} / \mathrm{m}^{3}$, lográndose un bajo potencial expansivo.

Finalmente, se presentan los resultados obtenidos para una arcilla con elevado potencial expansivo (arcilla tipo III), utilizando las mismas dosificaciones de aceite sulfonado. En la Figura 6 , se observa que el límite líquido se reduce de un valor inicial igual a $110 \%$ hasta valores alrededor de $80 \%$, suspendiéndose esta tendencia sin presentarse mayor diferencia entre las diferentes dosificaciones utilizadas; por el contrario, este parámetro vuelve a aumentar hasta quedar próximo al valor correspondiente a una arcilla no

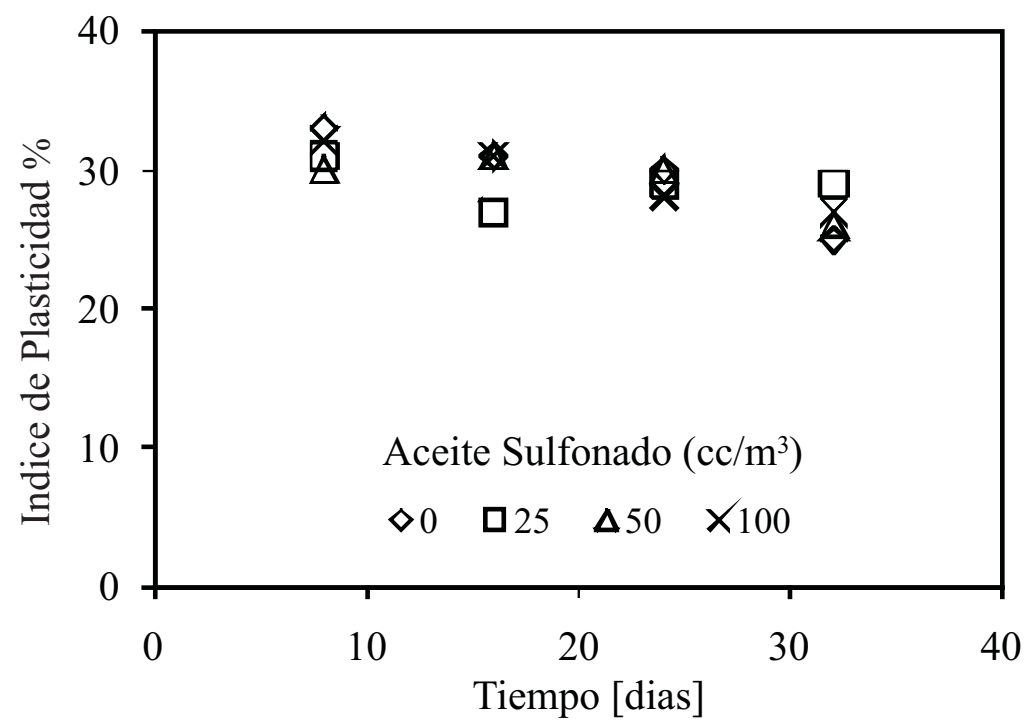

Figura 3. Variación del índice de plasticidad con el tiempo de exposición, para la arcilla tipo I.

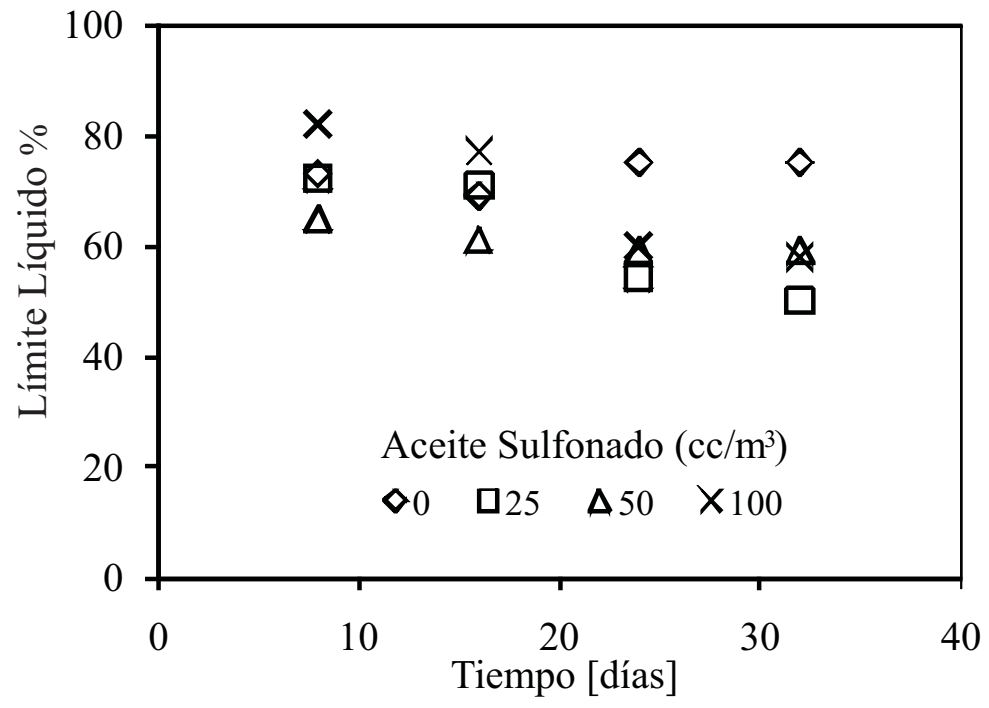

Figura 4. Variación del límite líquido con el tiempo de exposición, para la arcilla tipo II. 


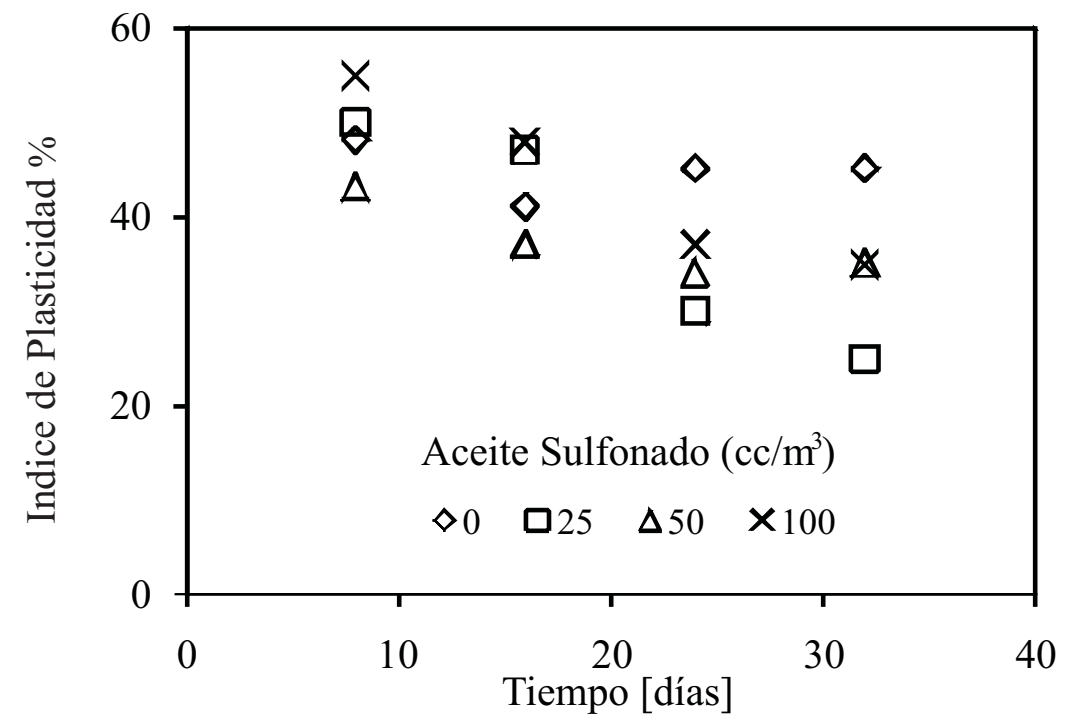

Figura 5. Variación del índice de plasticidad con el tiempo de exposición, para la arcilla tipo II.

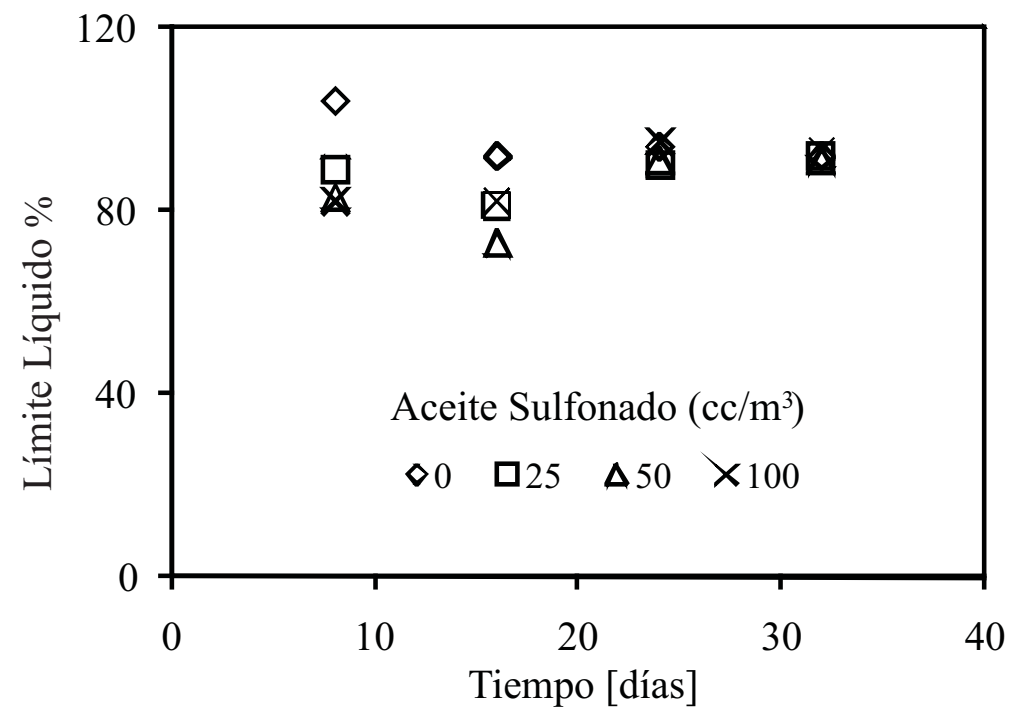

Figura 6. Variación del límite líquido con el tiempo de exposición, para la arcilla tipo III.

tratada. Este hecho se repite en la variación del índice de plasticidad (Figura 7).

Este comportamiento puede deberse a que la máxima dosificación utilizada es insuficiente para producir el efecto deseado sobre la arcilla expansiva. Esto comprueba una de las hipótesis formuladas al inicio del proyecto de investigación, referente a que la dosificación óptima de aceite sulfonado, depende del tipo de arcilla, por lo cual no se debería recomendar una dosificación única e independiente del tipo de material a tratar.
Las Figuras 8 y 9 sintetizan el efecto producido por diferentes dosificaciones de aceite sulfonado sobre los tres tipos de arcilla estudiados.

Se evidencia nuevamente que el límite líquido y el índice de plasticidad no sufren variaciones apreciables en los casos de las arcillas tipo I y tipo III, mientras que la arcilla tipo II si redujo su plasticidad como producto de la adición de aceite sulfonado.

El procedimiento utilizado en este estudio puede utilizarse para determinar las condiciones óptimas de utilización de aceite sulfonado, con respecto a la dosificación utilizada y el tiempo de curado en 


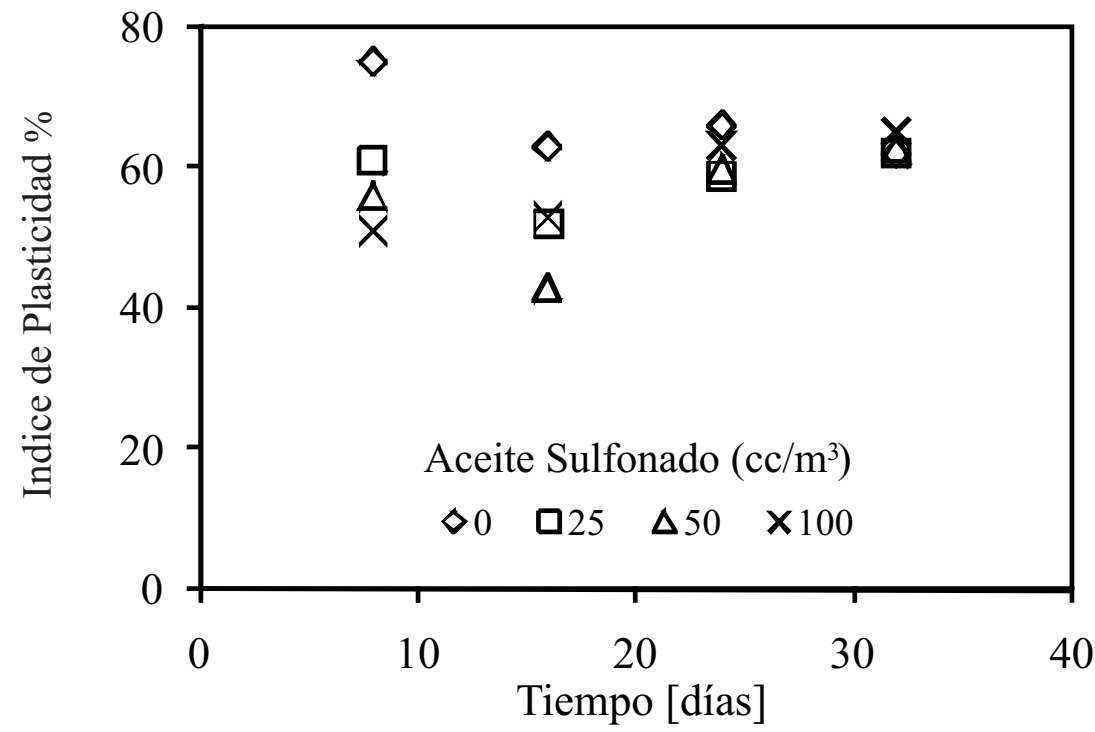

Figura 7. Variación del índice de plasticidad con el tiempo de exposición, para la arcilla tipo III.

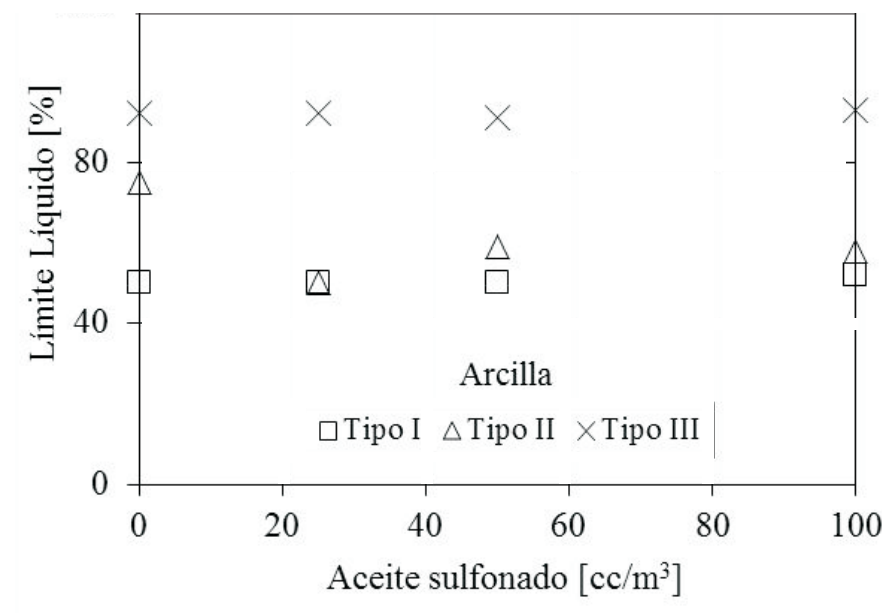

Figura 8. Variación del límite líquido la dosificación de aceite sulfonado.

relación al tipo de arcillas y las condiciones ambientales existentes en obra.

\section{Conclusiones}

Se simuló el proceso de curado natural de una arcilla estabilizada mediante aceite sulfonado, utilizando una cámara de envejecimiento acelerado cuya emisión de radiación UV actúa como acelerador de los procesos electroquímicos que se llevan a cabo entre el estabilizante, la arcilla y el agua intersticial. Este procedimiento puede servir para simular largos periodos de curado y estudiar tratamientos de estabilización y su efecto a largo plazo.

El proceso de curado analizado evidenció que existe una tendencia a la disminución de la plasticidad de una arcilla estabilizada con aceite sulfonado cuando es sometida a un proceso de curado bajo condiciones ambientales que permitan el desarrollo del proceso electroquímico.

No todos los tipos de arcilla reaccionan de la misma forma al tratamiento con aceite sulfonado y se observó que para arcillas altamente expansivas 


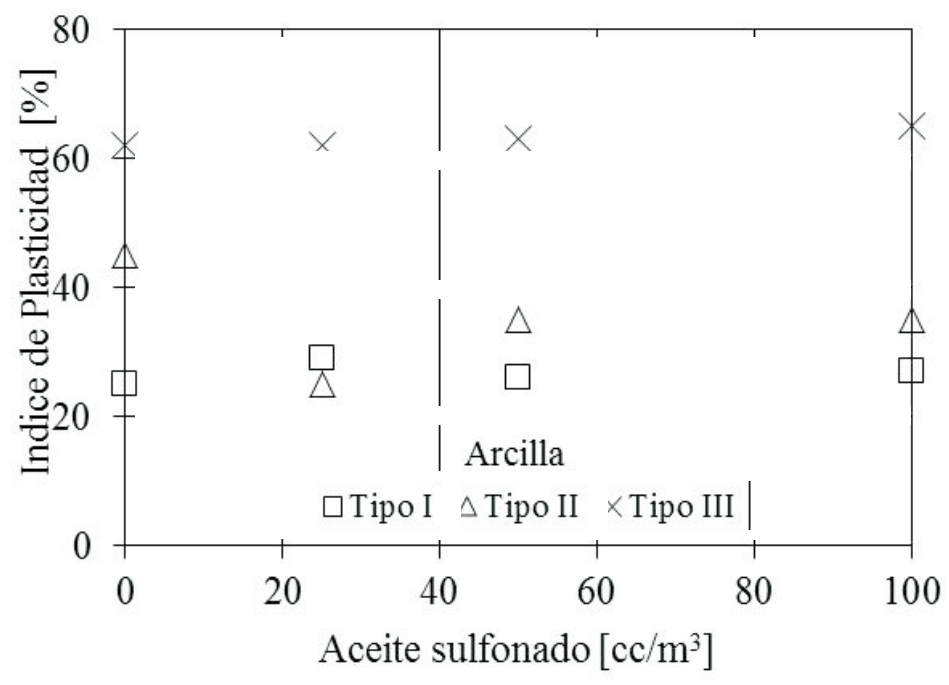

Figura 9. Variación del índice de plasticidad con la dosificación de aceite sulfonado.

los valores recomendados usualmente por el fabricante, no surten el efecto deseado, requiriéndose en estos casos un detenido análisis para obtener la mejor dosificación que reduzca efectivamente el potencial expansivo del material a niveles aceptables.

Se propuso una metodología para estudiar el uso del aceite sulfonado en el tratamiento de arcillas expansivas y obtener las dosificación óptima de acuerdo con las propiedades del material y la radiación UV a que está sometido durante el proceso de estabilización.

\section{Agradecimientos}

El presente trabajo se realizó dentro del proyecto de investigación denominado "Optimización del uso de Geo-Stab y metodología para su evaluación" desarrollado por el Grupo de Investigación Geotecnia de la Universidad Militar Nueva Granada y financiado por Colciencias, y Química Cros S.A. (Contrato 484-2005).

\section{Referencias Bibliográficas}

Arellana, J. (2004). Evaluación del efecto de la cal y el geostab en la estabilización de suelos arcillosos en Juan Mina; Barranquilla. Trabajo de Grado. Universidad del Norte; Facultad de Ingeniería Civil.

ASTM D 698 Standard Test Methods for Laboratory Compaction Characteristics of Soil Using Standard Effort. Pennsylvania, USA.

Camacho, J., Reyes, O., Mayorga, C. y Méndez, F. (2006). Evaluación de aditivos usados en el tratamiento de arcillas expansivas. Ciencia $e$ Ingeniería Neogranadina 16 (2). 45-53.

Day, R.W. (1994). Swell-shrink behavior of compacted clay. Journal of Geotechnical Engineering, ASCE, 120 (3). Technical Note No. 2740. 618-623.

Gadomski, C. (2007). Envejecimiento Acelerado. Ciclo conferencias Tecsud Ltda. Bogotá, Colombia.p.1-12.

Garzón, F., Diaz, C. y Páez D. (2004) Estabilización electroquímica con aceite sulfonado para arcillas representativas de la ciudad de Tunja. En: X Congreso Colombiano de Geotecnia. Sociedad Colombiana de Geotecnia. Paipa. 
Gens, A. y Alonso, E.E. (1992) A framework for the behaviour of unsaturated expansive clays. Canadian Geotechnical Journal. 29(6): 1013-1032.

IDEAM, (2009).

http://institucional.ideam.gov.co/jsp/loader.jsf?1Servi cio $\leq$ Publicaciones $\& 1$ Tipo $\leq$ publicaciones $\& 1$ Funcion $\leq$ loadContenidoPublicacion $\& \mathrm{id} \leq 273$ consultada en 02/12/2010.

IDEAM. (Unidad de Planeación Minero Energética e Instituto de Hidrología Meteorologia y Estudio Ambientales). (2005). Atlas de Radiación Solar de Colombia. Bogotá, Colombia.

INVIAS (Instituto Nacional de Vías). (2007) Normas de Ensayo para Materiales de Carreteras Vol Iy II. Bogotá, Colombia.

Kassiff, G. y Baker, R. (1971). Aging effects on swell potential of compacted clay. Journal of the Soil Mechanics and Foundations Division, ASCE, 97 (3), 529-540.

Mishra, A., Dhawan, S. y Rao, S. (2007). Analysis of swelling and shrinkage behavior of compacted clays. Geotechnical and Geological Engineering, 26(3), 289-298.
Páez Moreno, D. (2005). Efectos de la estabilización electroquímica de suelos finos. Revista Ingeniería UPTC, 14(18), 83-96.

Química Cros (2007).

http://www.acresquim.com/geo problem.htm

Rauch, A.F., Katz, L.E. y Liljestrand, H.M. (2003). An analysis of the mechanisms and efficacy of three liquid chemical soil stabilizers. Volume 1. Report No. FHWA/TX-03/1993-1. Texas Department of Transportation and the U.S. Department of Transportation.

Reyes, O. y Camacho, J. (2008). Efecto de la radiación ultravioleta en las propiedades mecánicas y dinámicas de una mezcla asfáltica. Revista ingeniería e investigación, 28 (3). 22-27.

Rodríguez H y González, F (1992). Manual de Radiación en Colombia, H. Rodríguez y F. González, Editores, Vol. II.

Subba, R y Tripathy, S. (2003). Effect of aging on swelling and swell-shrink behavior of a compacted expansive soil. Geotechnical Testing Journal 26(1). 36-45. 